\title{
The Walking Mileage Campaign: A Campaign Developed by an Information and Communication Technology (ICT)-based Program in the Workplace to Promote Walking by Making Donations to a Social Welfare Organization Based on Steps: A Pilot Study
}

\author{
Ritsuko Yamamoto-Honda' ${ }^{1)}$, Satoshi Sasaki ${ }^{2}$, Kyoko Kirii ${ }^{3,4)}$, Hiroyuki Mitsui ${ }^{5)}$, \\ Norio $\mathrm{Kano}^{5)}$, Mitsuhiko Noda ${ }^{1}$, Akihiro Igata ${ }^{6}$ \\ ${ }^{1)}$ Department of Diabetes and Metabolic Medicine and Diabetes Research Center, National Center for Global \\ Health and Medicine \\ ${ }^{2)}$ Department of Social and Preventive Epidemiology, School of Public Health, University of Tokyo \\ 3) Japan Foundation for the Promotion of International Medical Research Cooperation \\ ${ }^{4)}$ Department of Epidemiology and Prevention, National Center for Global Health and Medicine \\ ${ }^{5)}$ Eisai Co., Ltd \\ ${ }^{6)}$ Nagoya University of Arts and Sciences
}

\begin{abstract}
We present a novel motivational campaign (Walking Mileage) to promote continuous walking by making donations based on pedometer-recorded steps to a social welfare organization with the aid of Information and Communication Technology (ICT) solutions and devices. The aim of this report was to perform an age-related analysis of the participation rate, the persistence rate, and the daily steps taken as part of this campaign held in the workplace. Of the 1,254 participants (982 men and 272 women) recruited from 4,434 employees at a pharmaceutical company located in Tokyo, Japan, 1,124 completed the campaign, which lasted for $52 \mathrm{wk}$. Participants in their 50s tended to walk more and to have higher persistence rates than participants in their 40s, 30s, and 20s. The step counts for weekdays were larger than those for weekends and holidays.
\end{abstract}

Key words: health promotion in the workplace, pedometers, ICT-based

\section{* Background}

Walking accounts for much of the physical activity undertaken by middle-aged businesspeople. A pedometer is a useful motivational tool for encouraging an increase in walking ${ }^{1}$. An online pedometer pro-

Received: December 25, 2010

Accepted: December 8, 2011

Correspondence: M. Noda, Department of Diabetes and Metabolic Medicine and Diabetes Research Center, National Center for Global Health and Medicine, 1-21-1, Toyama, Shinjuku-ku, Tokyo 162-8655, Japan.

e-mail: mnoda@hosp.ncgm.go.jp gram is an effective tool for promoting continuous walking in local communities, with a participation rate of over $70 \%{ }^{2,3)}$. A donation to worthy causes based on the number of steps taken by the participants is another promising strategy for promoting walking in local communities ${ }^{4}$. Accordingly, we have proposed a novel motivational campaign (Walking Mileage) to promote walking by making donations based on pedometer-recorded steps to a social welfare organization through the use of Information and Communication Technology (ICT) solutions and devices. 
Table 1 Number of dropouts / participants at start point

\begin{tabular}{lcccc}
\hline & Age 20-29 & Age 30-49 & Age 40-49 & Age 50- \\
\hline Men & $29 / 68(42.6 \%)$ & $26 / 178(14.6 \%)$ & $20 / 369(5.4 \%)$ & $16 / 367\left(4.4 \%{ }^{+}\right)$ \\
Women & $31 / 59(52.5 \%)$ & $5 / 62(8.1 \%)$ & $2 / 76(2.6 \%)$ & $1 / 75\left(1.3 \%^{+}\right)$ \\
\hline
\end{tabular}

${ }^{+} \mathrm{p}<0.001$ among age groups according to the prop. trend. test.

Table 2 Median daily pedometer counts

\begin{tabular}{lcccc}
\hline & Age 20-29 & Age 30-49 & Age 40-49 & Age 50- \\
\hline Men & $6,796 *$ & $7,162 * * *$ & $7,468 * *$ & 8,521 \\
Women & $5,850 * * *$ & $6,672 * * *$ & $7,517 * *$ & 9,081 \\
\hline$* \mathrm{p}<0.05, * * \mathrm{p}<0.01, * * * \mathrm{p}<0.001$ & compared with the oldest age group (age over 50).
\end{tabular}

\section{Methods}

\section{Participants}

Data were collected from the records of employees of a pharmaceutical company based in Tokyo, Japan, who enrolled in the Walking Mileage campaign between November 1, 2006, and October 31, 2007. The study protocol was designed to comply with the Declaration of Helsinki. Informed consent was obtained from all the participants.

\section{Program}

Participants self-enrolled in the self-paced program via the Walking Mileage web site and updated their data by connecting their pedometer (HJ-7101T, Omron) to their personal computer. If the participants did not submit any data for $50 \mathrm{~d}$, they were regarded as dropouts from the campaign. Donations were made according to the number of steps taken by the participants. The rules of donation were as follows, where each point scored was equivalent to one Japanese yen $(¥)^{5)}$ : 1 ) daily steps from over 5,000 to $15,000,1$ point given for every 1,000 steps; 2 ) daily steps from over 15,000 to $25,000,1$ point given for every 2,000 steps; 3 ) over 70,000 steps per week, 3 additional points were given; 4) over 10,000 steps per $\mathrm{d}$ for more than 3 $\mathrm{d}$ per week, 1 point for the day with the largest number of steps. The Health Insurance Society underwrote the program cost and the cost of the donations.

\section{Aim of the study and statistics used in the present study}

The primary aim of this pilot study was to examine the persistent use of pedometers. We also examined the daily number of steps taken. All the data were analyzed using the R Project for Statistical Computing ${ }^{6}$.

\section{Results}

One thousand, two hundred and fifty-four participants were recruited into the study and 1,124 of them (89.6\%) completed the program. The participants wore their pedometers for $91.3 \%$ of the observation days and sent $93.8 \%$ of their daily step data. The older age group had a higher compliance and a larger number of daily steps with the program (Tables 1 and 2).

The pedometer counts were higher for weekdays and lower for weekends and holidays (Figure 1) as well as the rate of pedometer use (data not shown). The step counts declined during holiday periods (the New Year holidays, the so-called Japanese 'Golden Week' (which occurs at the beginning of May), and the summer holidays).

\section{Discussion}

The Walking Mileage 52-wk program was completed with a large number of participants and with few dropouts. The characteristics of this program could be suitable for workplace interventions which might offer a broader health benefit for employees 


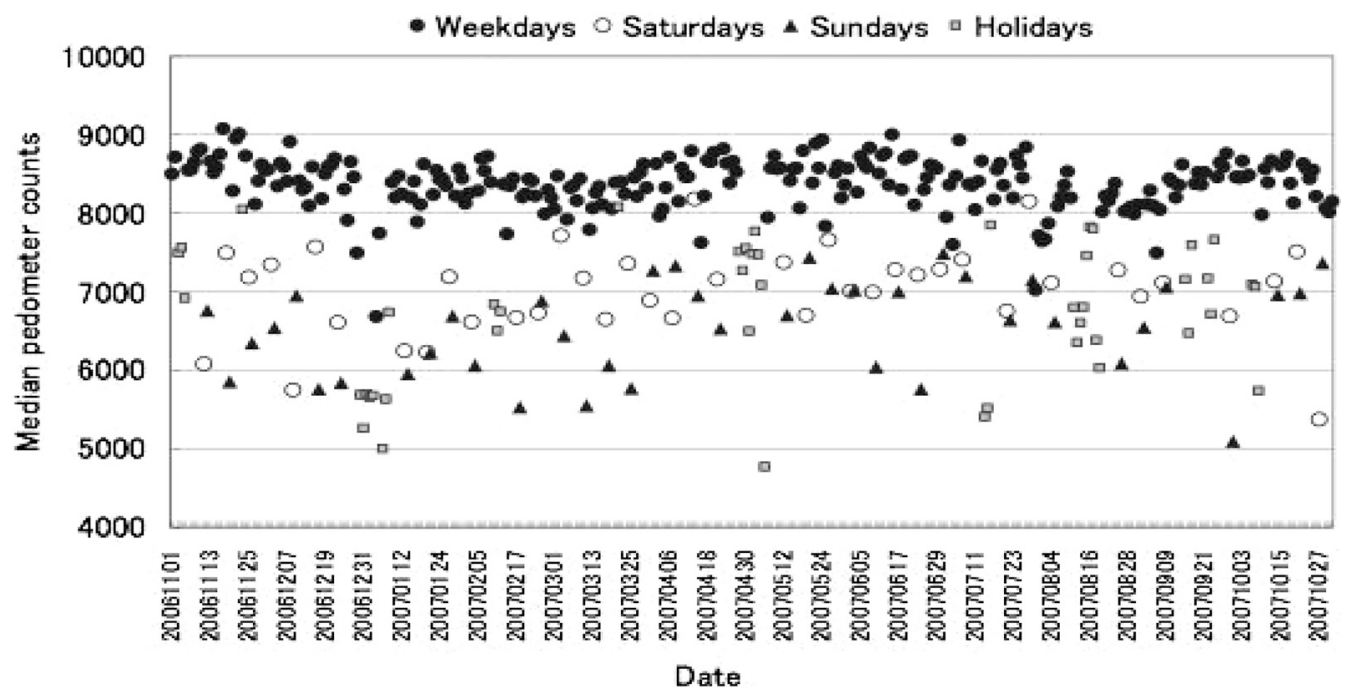

Figure 1 Daily profile of the median step counts of the participants.

Larger step counts were observed on weekdays than on Saturdays, Sundays, and holidays $(\mathrm{p}<0.001)$. Larger step counts were observed on Saturdays than on Sundays $(\mathrm{p}<0.01)$. The $\mathrm{p}$ value for the decreasing daily trend (by the prop. trend. test) was $<0.001$ for steps on weekdays. The $p$ value for the increasing daily trend (by the prop. trend. test) was $<0.001$ for steps on Sundays.

with a broad range of health statuses. In the next study, we would like to explore whether this strategy is effective for increasing pedometer counts and what kind of strategy is effective for increasing pedometer counts during holiday periods for young businesspeople.

\section{Acknowledgments}

This work was supported by a Health Science Research Grant (Research on Diabetes H18-002) from the Ministry of Health, Labour and Welfare of Japan. The authors would like to thank Eisai Co. and Eisai Health Insurance Society for participating in this work. The authors also sincerely thank Shigeru Tanaka (Graduate School of Business Administration, Keio University), Yoshiko Adachi (The Association for Preventive Medicine of Japan), Michio Matsuzaki (Matsuzaki Medical Clinic), and Hiroyuki Ishida (Sports Clinic, Department of Medicine, Keio University) for their valuable comments and advice.

\section{References}

1) Bravata DM, Smith-Spangler C, Sundaram V, et al.: Using pedometers to increase physical activity and improve health: a systematic review. JAMA 298, 2296-2304 (2007).

2) Booth AO, Nowson CA, Matters H: Evaluation of an interactive, Internet-based weight loss program: a pilot study. Health Educ Res 23, 371-381 (2008).

3) Jeffrey J, VanWormer MS, Nicolaas P, et al.: Experience analysis of a practice-based, online pedometer program. Diabetes Spectrum 19, 197-200 (2006).

4) Craig CL, Tudor-Locke C, Bauman A: Twelvemonth effects of Canada on the move: a populationwide campaign to promote pedometer use and walking. Health Educ Res 22, 406-413 (2007).

5) Kato M, Noda M, Igata A: The healthcare costsavings resulting from walking. The Asahi Shimbun January 23, 2010, http://ncgm-dm.jp/renkeibu/ hashin_4.html (access 13 August 2012).

6) R Development Core Team R: A language and environment for statistical computing. R Foundation for Statistical Computing, Vienna, Austria. http:// www.R-project.org (access 5 November 2010). 